\title{
The analysis and optimization of passenger security process

$$
\text { of terminal based on Monte Carlo model }
$$

\author{
Xinyue Tantai \\ School of North China Electric Power University, Baoding 071000, China; \\ 604798211@qq.com
}

Key words: Monte Carlo model

\begin{abstract}
Nowadays, passengers waiting in line in the security checkpoint always causes a high degree of congestion. In our paper, we find the bottleneck that interferes with passenger throughput, and propose solutions accordingly.First, the Monte Carlo model is established. And according to the Monte Carlo model, respectively, the conclusion that Zone $\mathrm{B}$ is the bottleneck of the security process can be obtained.Second, we optimize the above models. For the Zone B, one program is to set up waiting areas in the regular lanes. The regular passengers need to take out their belongings in it while the former one is being checked. Finally, the advantages and disadvantages of the model are evaluated and analyzed, and policies and procedure suggestions are put forward to optimize the airport security inspection process. What's more, the generalization of nonlinear comprehensive model is put forward.
\end{abstract}

\section{Monte Carlo Model}

\subsection{The Establishment of Monte Carlo Model}

We first analyzed the security work flow, as shown in Figure 1.

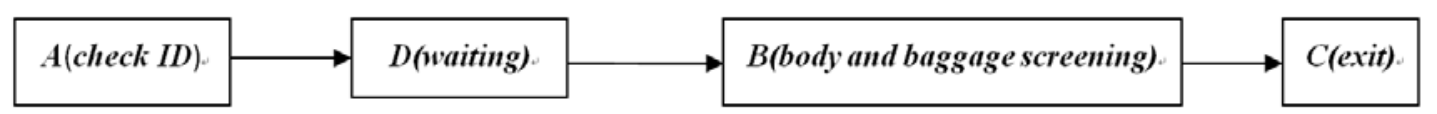

Figure 1 Security analysis process

Then, we determine the critical path of the security process: $A \rightarrow D \rightarrow B \rightarrow C$. Then we can use Monte Carlo simulation analysis to analyze the process. We mainly consider the critical path of all aspects of the check time and total time. In the security process,each link is discrete and random, and the simulation variables are the random factors $x_{i} \in\left(x_{1}, x_{2}, \cdots, x_{m}\right)$ that influence the check time of each link. The check time of each link is randomly chosen according to the negative exponential distribution. The 
ith link check time is denoted as $T_{k}\left(x_{i}\right)$. And the total time is equal to the sum of the check hours of each link in the critical routes. When the simulation runs for the $k$ th time, we get the total time $T_{k}$ of the $k$ th time, then $T_{k}\left(x_{i}\right)=T_{k}\left(x_{1}\right)+T_{k}\left(x_{2}\right)+\cdots+T_{k}\left(x_{m}\right)$; When the number of simulations is $\mathrm{N}$, let $T_{\text {min }}=\min \left(T_{k}\right), T_{\max }=\min \left(T_{k}\right), k \in(1,2, \ldots, N)$, and the total process time when it reaches steady state is the expectation $\mathrm{E}(\mathrm{T})$ of $\mathrm{N}$ times total simulation time.

First, we determine the number of simulation, set a single simulation run time $\mathrm{T}$ and $\mathrm{N}$ of the initial value. Initialization is performed at the beginning of a single simulation, and we determine the initial state $M_{0}$. Next, we execute the simulation process, and the simulation data is recorded after the process, and the above process is repeated until the preset simulation number is reached. Finally, the average check time $E\left(T\left(x_{i}\right)\right)$ and expected total time $\mathrm{E}(\mathrm{T})$ of each link are obtained from each simulation result.

According to the check time E (T (xi)), we can calculate the two important indicators of check time:

-The difference between the check hours $\Delta T\left(x_{i}\right)=T_{\min }\left(x_{i}\right)-T_{\max }\left(x_{i}\right)$

-The average check time in each link in the proportion of the total expected time $\frac{E\left(T\left(x_{i}\right)\right)}{E(T)}$

A link of the service time difference is large, indicating that this part of the service or operating time is not stable, and the impact on the stability of the system is big, and it is possible to make the entire passenger flow time longer; If the average service time of a link has a larger proportion of the total expected time, then it is the main part of the whole process, and it may be the bottleneck of the process.

\subsection{The Solution of Monte Carlo Model}

According to the above process of model building, through the simulation experiment, we obtained the two indicators of the pre-check and regular passengers in each zone, respectively, and the results are shown in Table 1. 
Table 1 The values of the two indicators

\begin{tabular}{ccc|cc}
\hline & & & $\Delta \mathrm{T}\left(\mathrm{x}_{\mathrm{i}}\right) / \mathrm{min}$ & $\mathrm{E}\left(\mathrm{T}\left(\mathrm{x}_{\mathrm{i}}\right)\right) / \mathrm{E}(\mathrm{T})$ \\
\hline \multirow{3}{*}{ Pre-check passengers } & Zone & $\mathrm{A}$ & 0.8979 & $0.8617 \%$ \\
& Zone & $\mathrm{B}$ & 59.4628 & $94.4181 \%$ \\
& Zone & $\mathrm{C}$ & 4.3199 & $4.7202 \%$ \\
\hline \multirow{3}{*}{ Regular passengers } & Zone & $\mathrm{A}$ & 1.4377 & $0.5289 \%$ \\
& Zone & $\mathrm{B}$ & 40.2456 & $96.6842 \%$ \\
& Zone & $\mathrm{C}$ & 6.7394 & $2.7869 \%$ \\
\hline
\end{tabular}

From Table 1, we can see, in Zone B, the check time difference and the average check time in the expected proportion of the total time of the pre-check and regular passengers are large. It indicates that the inspection in the B area is a weak link. Thus, Zone B makes a big difference on the stability of the system, and it is the main link for the entire system. So we determine B is the bottleneck

\section{Model optimization}

From the above Monte Carlo model, we can see that the problem of regular lanes in Zone B is the biggest. According to the data given by the table, we can see that the time regular passengers spend most in the Zone B is the process of removing their items. So we set up a waiting area in Zone B for the passengers to remove their items. And after receiving the inspection in Zone A, regular passengers immediately enter the waiting area. In this case, we consider the process that regular passengers accept the inspection in Zone B is the same as the pre-check passengers.

In order to describe this improvement better, we made a model diagram. As is shown in Figure 2.

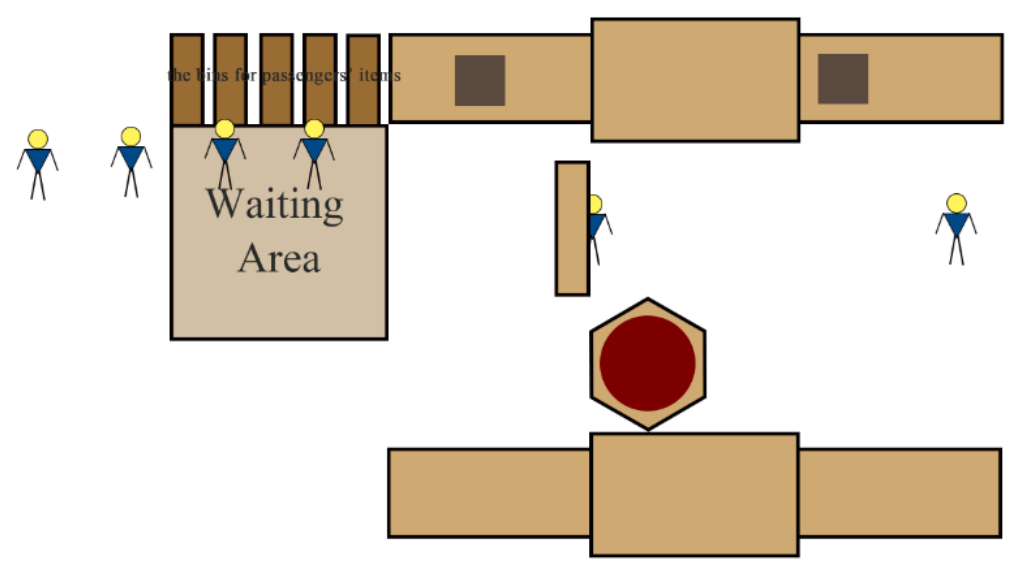

Figure 2 The model with waiting area 


\section{Conclusion}

In order to improve the accuracy of Monte Carlo simulation, we should find more data and refine the security process.

In addition, the impact of cultural factors on the improvement of the program can also be analyzed with the analytic hierarchy process, fuzzy evaluation, neural networks and other methods. But on the whole, each model including Monte Carlo simulation model has different degree of limitation. But we cannot deny that each model also has its own advantages. Therefore, we can consider establishing nonlinear integrated model to combine various types of models to avoid weaknesses, thus getting more desirable results.

Furthermore, the security process is essentially a complex system of passengers, staff, inspection machines, airport service situation, which itself has a non-linear interaction. Moreover, the internal process of the system is irreversible, and the use of non-linear comprehensive model is very effective to solve these problems. Therefore, the comprehensive model based on the nonlinear system theory will have a wide application prospect for the optimization and evaluation of security process.

\section{References}

[1]Guoyang Jia. Study on Optimization of Terminal Passenger Screening Service Process Based on LCIOWF [D].Harbin Institute of Technology,2015.

[2]Xun Lu. Research on the Planning and Simulation of Passenger and Luggage Process at Airport Landside [D]. Nanjing University of Aeronautics and Astronautics,2008.

[3]Shoukui Si, Xijing Sun. Mathematical Modeling Algorithm and Program, Beijing: National Defense Industry Press, 2011

[4]LAX flight. Airport departures from 9:00 to 10:00 on January 22nd, http://www.airport-la.com/lax/departures?t=3 